\title{
Bioanalysis
}

\section{Clinical and pharmaceutical success from discovery to regulatory approval: biomarkers, modeling and analytical technologies}

\begin{abstract}
The 8th Annual Shanghai Symposium on Clinical and Pharmaceutical Solutions through Analysis (CPSA): Clinical and Pharmaceutical Success from Discovery to Regulatory Approval: Biomarkers, Modeling and Analytical Technologies (CPSA Shanghai 2017); Renaissance Shanghai Pudong Hotel, Shanghai, China, 12-14 April 2017
\end{abstract}

The meeting was featured with highly interactive events including diversified symposia, workshops, roundtable discussions, conference awards and poster sessions. There were over 220 participants with 61 oral presentations and 20 posters presented. In addition, the meeting included a preconference workshop with an inaugural session on the evaluation of quality and efficacy for generic drugs in China.

First draft submitted: 11 July 2017; Accepted for publication: 18 August 2017;

Published online: 21 October 2017

Keywords: biomarker $\bullet$ biomolecule $\bullet$ DMPK $\bullet$ drug development $\bullet$ generic drug $\bullet$ LC-MS bioanalysis $\bullet$ quality and efficacy evaluation

\section{CPSA Shanghai 2017 program}

Nathan Yates of University of Pittsburgh (PA, USA) served as the CPSA Shanghai 2017 Program Chair. The theme of this year's annual meeting was 'Clinical and Pharmaceutical Success from Discovery to Regulatory Approval: Biomarkers, Modeling, and Analytical Technologies' [1]. The CPSA founder, Mike Lee of Milestone Development Services (PA, USA) greeted the audience. Renuka Pillutla of Bristol-Myers Squibb (NJ, USA) delivered a keynote speech on 'Getting More Drugs to Patients Faster: the Power of Technology Applications'. It was noted proteomics could measure age-related changes in biological species. Proteomic data can be used as biomarkers to promote age modeling and anti-aging drug development in future clinical and diagnostic trials.

Cornelis Hop of Genentech (CA, USA) gave a plenary lecture on 'Drug Discovery and Development: Looking for Needles in a Haystack'. From the 1900s to the pres- ent, human disease spectrum has undergone notable changes, and people's demand for medical treatment has also changed, which presents new challenges for drug $R \& D$. To address the challenges, it is necessary to pursue a clear understanding of the mechanism of drug action, the relationship between PK/ PD and efficacy, and the choice of clinical PD biomarkers.

The symposium included 16 sessions covering a broad range of topics. This conference report summarizes some highlights from the contributed sessions.

\section{Evaluation of quality \& efficacy for generic drugs in China}

This session was chaired by Tianyi Zhang of Frontage Laboratories (Shanghai, China) and Jeff Zhang of Novartis (Shanghai, China). This was a special inaugural session addressing the ongoing initiative from the China FDA (CFDA). Historically, generic drugs in China were required to show con-
Tianyi (Tee) Zhang ${ }^{*, 1}$, Naidong Weng ${ }^{2} \&$ Mike Lee $^{3}$ ${ }^{1}$ No.13 Building, 67 Libing Road, Zhangjiang Hi-Tech Park, Shanghai, China 201203, PR China 2Janssen, Pharmaceutical companies of Johnson \& Johnson, 1400 McKean Road, Spring House, PA 19477, USA ${ }^{3}$ Milestone Development Services, 7 Snowdrop Place, Newtown, PA 18940, USA

*Author for correspondence: tianyizhang@frontagelab.com.cn 
formity with the national standards but not required to undertake bioequivalent (BE) studies against originator drugs. As a result, the generic drugs are not deemed equivalent to the originator drugs. Currently CFDA has launched a consistency evaluation of quality and efficacy for generic drugs in China by using originator drugs as reference, aiming to enhance the quality of generic drugs. As a result, drug manufacturers are required to submit data to demonstrate equivalence of the generic drugs with the originator drugs. Clinical bioequivalent studies (BE) are the primary method used in the evaluation.

Tianyi Zhang presented 'How to Conduct Clinical Bioequivalence Study and Related Bioanalysis'. The key considerations in a BE clinical study include selection of reference drugs, design of clinical protocol, clinical study conduct and study monitoring, and the performance of bioanalysis, PK and statistical analysis, etc. The outcome of the BE study relies on three most important factors: drug formulation, clinical conduct and bioanalytical measurement, which determine the outcome of the BE study.

Jun Fan of Overseas Pharmaceutical (Taizhou, China) presented 'Formulation Development Related to BE Study'. The generic drug must show pharmaceutical equivalence with the originator drug via in vitro dissolution test. Stability test of the generic drug formulation is also required. Before a pivotal BE study is launched, a pilot study with fewer subjects is recommended.

Minji Wei of Peking University (Beijing, China) presented on 'Regulatory Requirement and Inspection with BE Study'. Since July of 2015, CFDA has launched a heavy-handed inspection for clinical studies and bioanalytical laboratories. Over $80 \%$ generic drug applications were withdrawn from the CFDA review and approval system due to data integrity concern. The report discussed the regulatory requirements and how CFDA inspectors would inspect a clinical BE study.

\section{Hot topics in Drug Metabolism and Pharmacokinetics (DMPK)}

This session was chaired by Naidong Weng of Janssen Research \& Development (Spring House, PA, USA). Six speakers presented a range of hot topics on current DMPK development.

Sophia Xu of Bristol-Myers Squibb (NJ, USA) presented 'Full Measurement of Highly Protein-Bound Drugs'. In the human body, unbound (fu) concentrations are very important in therapeutics because unbound drugs can pass through most cell membranes and equilibrate with the extra-vascular space. Unbound drug is more closely related to the pharmacologic effect. The use of various techniques including gold standard format, equilibrium dialysis, ultrafiltration and ultracentrifugation to measure fu concentration was discussed.

Shaolian Zhou of Roche (Basel, Switzerland) reported 'Bioanalytical Strategy for Metabolites in Safety Testing (MIST) Coverage: Human Unique Metabolites'. The key implication of the new US FDA guidance Safety Testing of Drug Metabolites Guidance for Industry and its impact on bioanalytical practice were discussed. New approaches of measuring human metabolites and bioanalytical strategy for MIST coverage were illustrated in several case studies.

Shuguang Ma of Genentech (CA, USA) presented on 'Microdosing/Microtracers in Clinical Development'. Bioavailability (BA) is a measurement of the rate and extent to which a drug's active moiety or active ingredient is absorbed, reaches the systemic circulation and becomes available at the site of action. For new chemical entities, absolute BA in humans is increasingly requested by the FDA, the EMA and Japanese regulatory agencies, and since 2006 has been required for new drug application submissions to Australian Therapeutic Goods Administration. The clinical study design to obtain absolute BA data for the registration of pharmaceuticals has been opened up and simplified by the microtracer approach.

Jialin Mao and Yuan Chen of Genentech (CA, USA) presented 'Physiologically Based Pharmacokinetics (PBPK) Application in Drug Development'. Much attention was given to PBPK modeling by the pharmaceutical industry and regulatory agencies in the past 5 years. Both US FDA and EMA published the draft guidance on the PBPK modeling for drug development. The presentation provided a comprehensive review on the following: the view from the regulatory agency on PBPK; the view from the pharmaceutical industry: the impact of PBPK in drug discovery and development; and the future of PBPK.

Keyang Xu of Genentech (CA, USA) presented 'Bioanalytical and Biotransformation of Antibody Drug Conjugate (ADC) Drugs'. The presentation described the complexity of ADCs in vivo and different types of assays currently available for the $\mathrm{PK}$ and biotransformation evaluation of ADC. A couple of case studies were presented to illustrate the fit-for-purpose bioanalytical assay strategies.

Ming Yao of Bristol-Myers Squibb (NJ, USA) presented 'Fast and Sensitive Quantitation of Low Levels of Intracellular Substrates in Uptake Transporter Assays using Liquid Chromatography Mass Spectrometry'. The determination of transporter substrate in vitro is common, and often employs radio-labeled compounds to achieve high analytical sensitivity. This presentation reported the development of a generic, 
sensitive LC-MS methodology for substrate analysis of uptake transporter experiments using atorvastatin and olmesartan as two model compounds.

\section{Paradigm shift in global \& bioanalysis support in China}

In an Executive Roundtable discussion chaired by Naidong Weng and Ajai Kumar Chaudhary of Merck (NJ, USA), a group of panelists including Renuka Pillutla, Cornelis Hop, Shaolian Zhou, Kelly Dong of GlaxoSmithKline (Shanghai, China), Xinping Fang of Wuxi AppTec (Shanghai, China), Tianyi Zhang and Jin Fan of Covance (Shanghai, China) discussed the paradigm shift in global and local bioanalysis requirements. Low cost and on-time delivery in a more complicated regulatory environment remains to be a major incentive for pharma to outsource bioanalytical work. The concepts of scientific validation versus tiered approach versus fit-for-purpose were discussed.

The topics of whether we have the right bioanalytical infrastructure between the large and small molecules drug development/bioanalytical service groups, how we fully explore the potential of new instrumentation, how we manage the regulatory environment globally and locally, for example, in China, where global and local CROs view their strategies to support global and local pharma in the future attracted lots of interesting discussions from the panelists and audiences.

\section{Innovative approaches to drug development}

This session was chaired by Shaolian Zhou and Sylvia Zhao of Novartis (Shanghai, China).

Naidong Weng presented 'Labeled Isotope Compounds in LC-MS Based Bioanalysis'. For small molecules, labeled compounds can be used in their biological analysis without the addition of internal standard substances. Trace-labeled compounds can be used for the determination of absolute BA in the human body. They can also be used for quantification of drug metabolites and can be used as biomarkers for the analysis of alternative substances.

Jian Xu of Novartis (Shanghai, China) presented 'Clinical Pharmacology Impact on the Recommended Phase II Dose Selection in Immuno-oncology'. More than $90 \%$ of the candidate drugs for Phase I clinical trials end in failure. For oncology drugs, over $73 \%$ failure occurred in Phase II due to inadequate efficacy and safety indications.

Keyang Xu presented 'Innovative LC-MS Applications in Characterization and Quantification of Biologics In vivo: Challenges and Opportunities'. The complexity of antibody-coupled drugs poses a great challenge to their biological analysis. The change of $\mathrm{ADC}$ in vivo has a great influence on its safety and effectiveness. A variety of analytical methods should be used to ensure measurement quality during the development of ADC drugs. Use of LC-MS method to measure ADC drug metabolites was reported to be a future trend.

\section{Biomarker challenges \& biomolecule bioanalysis}

This session was chaired by Wenying Jian and Naidong Weng and Qin Ji of Bristol-Myers Squibb (NJ, USA). Yang Qiu of Janssen (PA, USA) presented 'Biomarker for Patient Selection'. Many molecular targets are reliable markers for selecting patients to improve treatment effectiveness. The report discussed important considerations and challenges in transferring biomarker assay information into clinically actionable decisions to help with disease diagnosis and personalized medicine.

Daniel Sikkema, Frontage Laboratories (PA, USA) presented on 'Complexities and Solutions for Biomarker Support of an Increasingly Complex Drug Development Portfolio'. The report discussed a variety of challenges for biomarker measurement, and proposed innovative solutions with new instrumentation technology to develop highly sensitive and selective assays for biomarker determination.

Wenying Jian of Janssen Research \& Development (PA, USA) presented 'Liquid Chromatography-High Resolution Mass Spectrometry (LC-HRMS) Based Bioanalysis of Intact Proteins'. LC-HRMS is a powerful analytical tool used to determine intact proteins. The report discussed strategies utilizing highresolution mass spectrometry in LC-MS to improve specificity for biomarker quantitation.

Song Zhao of Frontage Laboratories (Shanghai, China) presented 'LC-MS/MS for Large Molecule Bioanalysis'. LC-MS/MS is especially powerful for antibody-drug-conjugate determination. The report presented the challenges and strategies for utilizing LC-MS/MS for large molecule bioanalysis.

\section{Hot topics of bioanalysis}

There were two sessions with six presenters to cover a range of hot topics in bioanalysis. One session was chaired by Sophia Xu and Frances Wang of Janssen (Shanghai, China). The other session was chaired by Tianyi Zhang and John Ling of Gilead Sciences (CA, USA).

Qikuan Huang of WuXi AppTec (Shanghai, China) presented 'Key Considerations for the Regulated Immunogenicity Assessment in Support of Clinical Trials'. Pre-existing antibodies and other interfering matrix components may impact immunogenicity assay cut-point determination and cause false-negative 
results for patients. Strategies for mitigating the impact of these factors were discussed.

John Ling presented 'Commonly Asked Bioanalytical Questions by FDA, EMA, Health Canada (TPD) and Japan PMDA'. The perspectives of the bioanalytical guidances from different regulatory agencies were discussed. Several case studies were presented, with the common findings and commonly asked questions from these agency representatives shared in the presentation.

Tianyi Zhang presented 'Clinical BE Studies and Related Bioanalysis Requirements in China'. BE study is the primary method recommended by CFDA for the evaluation of quality and efficacy for generic drugs in China. The BE success depends on several critical factors including drug products, clinical protocol design and study conduct, and accuracy of bioanalytical measurement, etc. The report discussed some key considerations and requirements for clinical BE studies and bioanalysis in China.

Jing Tu of PPD Inc. (VA, USA) presented 'Challenges in Developing and Validating a Biomarker/ Endogenous Ligand-Binding Assay (LBA) following GLP Guidelines'. The report discussed the current challenges in biomarker measurement and recommendations for using surrogate matrix following a fit-forpurpose approach for biomarker validation. Determination of soluble B-cell maturation antigen (sBCMA) in human serum following a fit-for-purpose approach was illustrated during the presentation.

B-cell maturation antigen (BCMA

Sophia $\mathrm{Xu}$ presented 'Bioanalytical Challenge of Measuring Highly Protein Bound Drugs'. Measurement of protein-bound drugs can be challenging, due to many factors including assay sensitivity, nonspecific binding and unable to reach equilibrium. The report illustrated several strategies for measuring highly bound drugs using several techniques such as partitioning, ultrafiltration and equilibrium dialysis, etc. Inaccurate protein-binding measurement may impact the observed versus predicted human PK results such as clearance rate, volume of dilution and elimination half-life time, etc.

Min Meng, an independent consultant (Chongqing, China), presented 'Conducting Bioequivalence Studies in China: Challenges and Solutions'. In this presentation, the systematic and innovative strategy

\section{References}

1 CPSA Shanghai 2017. www.cpsa-shanghai.com/2017/index.shtml was elucidated using several case studies. The impact of phospholipids on the assay performance and ways to remove phospholipids were discussed.

\section{Posters \& conference awards}

There were a total of 20 posters, focusing on bioanalytical issues and solutions, first-hand experiences with specific applications and technologies shared by young scientists. The CPSA Shanghai meeting attracted many young scientists and researchers. To recognize their work and poster presentations, the meeting has implemented the Young Scientist Excellence Award and Innovator Award since 2012.

\section{Summary}

The 8th CPSA Shanghai conference has presented diverse topics of interest for drug researchers, focusing on the clinical and pharmaceutical success from discovery to regulatory approval. As in the past, the conference was featured with highly interactive and diversified events, and has attracted attendees from overseas and local communities. Of particular interest was the addition of an inaugural session on the ongoing quality and efficacy evaluation for generic drug products in China, which has significant impact on the pharmaceutical industry and CRO services in China. Biomarkers, PK/PD modeling and analytical technologies with new applications have presented new means for providing insightful data in drug research. This conference report summarized the key events at the conference to promote learning and sharing. Plans are underway for next year's conference, CPSA Shanghai 2018. For more details please visit the conference website [2].

Financial \& competing interests disclosure

The authors have no relevant affiliations or financial involvement with any organization or entity with a financial interest in or financial conflict with the subject matter or materials discussed in the manuscript. This includes employment, consultancies, honoraria, stock ownership or options, expert testimony, grants or patents received or pending, or royalties.

No writing assistance was utilized in the production of this manuscript.

2 CPSA Shanghai 2018. www.cpsa-shanghai.com. 